\title{
Axial distribution of deep-level defects in as-grown CdZnTe:In ingots and their effects on the material's electrical properties
}

\author{
Lingyan Xu ${ }^{\text {a }}$, Wanqi Jie ${ }^{\mathrm{a}, *}, \mathrm{Xu} \mathrm{Fu}{ }^{\mathrm{a}}$, A. E. Bolotnikov ${ }^{\mathrm{b}}$, R. B. James ${ }^{\mathrm{b}}$, Tao Feng ${ }^{\mathrm{a}}$, Gangqiang Zha ${ }^{\mathrm{a},+}$, \\ Tao Wang ${ }^{\text {a }}$, Yadong $\mathrm{Xu}^{\mathrm{a}}$, and Yasir Zaman ${ }^{\mathrm{a}}$ \\ ${ }^{a}$ State Key Laboratory of Solidification Processing, School of Materials Science and Engineering, \\ Northwestern Polytechnical University, Xi'an, Shaanxi 710072, China \\ ${ }^{\mathrm{b}}$ Brookhaven National Laboratory, Upton, NY 11973, USA
}

\begin{abstract}
We investigated the distribution of deep-level defects in CdZnTe:In ingots along the growth direction using thermally stimulated current (TSC) spectra. Higher concentration of Te antisite-related deep donors were found in the tail region due to Te enrichment in the melt as solidification proceeds. The compensation between $\operatorname{In}_{C d}^{+}$defects and $\mathrm{Cd}$ vacancies results in low concentration of net free electrons. High resistivity with n-type conduction was demonstrated from the I-V analysis. Hall mobility is lower in the tail due to higher concentration of Te antisite-related deep donors.
\end{abstract}

\section{Keywords}

B2. Semiconducting II-VI materials, A2. Bridgman technique, A1. Defects, A1. Characterization

Corresponding author: Tel.: +86-13772439913; Fax: +86-29-88495414;

E-mail address: xulingyan1987@gmail.com.

\section{Introduction}

Cadmium zinc telluride (CdZnTe, CZT) crystals have been considered as one of the most promising 
compound semiconductors for the applications as room-temperature X-ray and $\gamma$-ray detectors [1]. Although the extensive usage of this material due to its excellent opto-electrical properties, the quality of this material needs to be further optimized [2]. As-grown CZT crystals generally encounter problems arising from the bulk defects and the non-uniformity of elemental distribution, which can significantly degrade the crystal properties and consequently deteriorate the performances of detectors [3, 4]. Cadmium vacancies have been considered to be the dominant native defects and responsible for the p-type conductivity of high-purity undoped CdTe and CdZnTe crystals, due to the high partial pressure of Cd under non-stoichiometric conditions during crystal growth [5]. Group III (Al, Ga, In) or group VII $(\mathrm{Cl}, \mathrm{Br})$ elements incorporating on $\mathrm{Cd}$ sites can introduce donor levels to compensate $\mathrm{Cd}$ vacancies (acceptors) [6]. Under tellurium-rich conditions, the maximum solubility of excess Te in CZT crystals at $\sim 860{ }^{\circ} \mathrm{C}$ is about $4 \times 10^{18} \mathrm{~cm}^{-3}$. The retrograde solubility leads to Te precipitation at lower temperature as well as Te antisites (Te atoms on Cd sites), Te interstitials, Cd vacancy-Te antisite pairs and so on [7,8]. These defects introduce energy levels in the bandgap, which play a dual role in both the compensation of defects and the trapping of generated charge carriers. Deep-level defects are responsible for high resistivity by stabilizing the compensation conditions [9]. However, at the same time, they interfere with the carrier transport process and cause incomplete charge collection, acting as recombination and trapping centers [10]. Moreover, the de-trapping of the trapped carriers would induce both polarization and after-glow effects, especially under high-flux radiation environment $[11,12]$. In this study, we investigated the size distribution and concentration of Te inclusions along the growth direction, correlated with the process of crystal growth. The distribution of deep-level defects was studied using thermally stimulated current (TSC) measurements. The effects of these defects on the electrical properties were discussed to better 
illuminate the nature of the defects' behaviors.

\section{Experimental}

CdZnTe single crystals, with the dimension of $10 \times 10 \times 1 \mathrm{~mm}^{3}$, were cut axially from the tip (first-to-freeze), middle, and tail (last-to-freeze) regions of as-grown $\mathrm{Cd}_{0.9} \mathrm{Zn}_{0.1} \mathrm{Te}$ :In ingots grown by the Modified Vertical Bridgman (MVB) method. The size distribution and concentration of Te inclusions in CZT crystals were investigated using infrared (IR) transmission microscopy at Brookhaven National Laboratory (BNL). Due to inclusion scattering of IR light, we can visualize both inclusions, with diameters down to 1-2 $\mu \mathrm{m}$, and inclusion-related extended defects from the contrast of IR images. We utilized thermally stimulated current (TSC) measurements to investigate the energy distribution of trap levels in the band-gap, which are associated with defect states in the crystal lattice. At low temperature, free carriers excited by achromatic light from a halogen lamp will be captured by defect traps. As the temperature increases in the dark from 80 to $320 \mathrm{~K}$ at a constant heating rate, trapped electrons and holes released by thermal emission are scanned and recorded under an applied bias to form the thermally stimulated current. An Agilent 4155C setup was employed for current-voltage (I-V) analysis to assess the electrical contact and to determine the bulk resistivity. Carrier mobility and conduction type were detected by means of Van der Pauw method based on Hall effect.

\section{Results and Discussion}

A Te-rich boundary layer will be formed in the melt due to the limited mixture during the growth process of CdZnTe crystals, which may be captured by the growth interface, thus forming Te inclusions $[13,14]$. Fig. 1 shows IR microscopic images with size distribution and concentration of Te inclusions in CdZnTe wafers along the growth direction of the as-grown ingots. Small inclusions (1-10 $\mu$ m) with 
average concentration of $\sim 10^{4} \mathrm{~cm}^{-3}$ and big ones (>10 um) with average concentration of $\sim 10^{3} \mathrm{~cm}^{-3}$ were detected in all the samples. The largest size of Te inclusions in CdZnTe wafers from the tip region is about $16 \mu \mathrm{m}$, and that in the tail is about $33 \mu \mathrm{m}$. The distribution of Te inclusions along the growth direction of as-grown CdZnTe ingots could be ascribed to the combined effect of multiple factors, e.g., the furnace's temperature field and the growth rate $[4,15]$. Generally, excess $\mathrm{Te}$ in the non-stoichiometric melt becomes enriched gradually as solidification proceeds, which would probably cause a greater number of bigger Te inclusions in the tail. Moreover, Te enrichment in the melt in front of the growth interface leads to an increase in the morphological instability, and a rise in the density of native point defects (e.g., Cd vacancies) along the growth direction [4].

Thermally stimulated current (TSC) measurements were used to investigate deep-level defects in CZT crystals. Each peak in TSC spectrum corresponds to a certain trap level in the band-gap, and a certain defect state in the crystal lattice. Trap-related parameters, e.g., the activation energy $E_{T}$, the capture cross-section $\sigma_{T}$ and the trap density $N_{T}$ were obtained. In the "first-order kinetics" approximation, the temperature $T$-dependent TSC current $I_{T S C}$ of a certain peak could be described as [16-18]:

$$
I_{T S C}(T)=K_{G} \mu \tau N_{T} D_{t} T^{2} \exp \left\{-\frac{E_{T}}{k_{0} T}-\frac{k_{0} D_{t} T^{4}}{\beta E_{T}} \exp \left(-\frac{E_{T}}{k_{0} T}\right)\left[1-4\left(\frac{k_{0} T}{E_{T}}\right)+20\left(\frac{k_{0} T}{E_{T}}\right)^{2}\right]\right\}
$$

where $\mu \tau$ is the mobility-lifetime product of the charge carriers. $K_{G}=e A E$ is the geometrical factor, where $e$ is the electron's charge, $A$ is the electrode's area, and $E$ is the strength of the electric field. The trap-dependent coefficient is defined as $D_{t}=3.0 \times 10^{21}\left(\mathrm{~m}^{*} / m_{0}\right) \sigma_{T}$ [18]. The effective masses of electrons and holes are $m_{e}^{*}=0.14 m_{0}$ and $m_{h}^{*}=0.37 m_{0}$, with $m_{0}$ being the electron's rest mass [19]. $k_{0}$ is the Boltzmann's constant, and $\beta$ is the heating rate. 
Fig. 2 shows typical TSC spectra of CdZnTe wafers from the tip, middle, and tail samples of the as-grown ingots. The classical method of single peak analysis was not suitable to precisely determine trap signatures in TSC spectra due to the mutual interference from overlapping peaks. Hence, the simultaneous multiple peak analysis (SIMPA) method was adopted [18]. Through SIMPA fitting, we found four main trap peaks (T1 T4) in all the samples. The level labeled T1 has an activation energy of $0.06 \pm 0.01 \mathrm{eV}$ below the conduction band minimum, with the trap type (electron or hole) revealed by thermoelectric effect spectroscopy (TEES) [20, 21]. T1 could be assigned to a shallow donor originated at In dopant related point defect $\operatorname{In}_{C d}^{+}[22,23]$. Traps T2 and T3 are recognized as acceptor defects, located at $0.11 \pm 0.01 \mathrm{eV}$ and $0.21 \pm 0.01 \mathrm{eV}$ above the valence band maximum, respectively. The microscopic origins of these two traps are attributed, respectively, to the firstly ionized and secondly ionized Cd vacancies $[23,24]$. Trap T4 located at $0.56 \pm 0.02 \mathrm{eV}$ below the conduction band minimum could be attributed to a deep donor Te antisite $\left(T e_{C d}^{2+}\right.$ ) with the second ionization energy of $\sim 0.59 \mathrm{eV}$ according to theoretical calculations [23].

Melt enrichment of Te leads to higher densities of Te-rich phases and Cd vacancy defects in the tail region compared with that in the tip, as discussed above. Oversaturated Te atoms are likely to occupy the positions of $\mathrm{Cd}$ vacancies and to form Te antisites [8, 25]. Accordingly, the trap density of Te antisite-related deep donors (T4) is higher in the tail than that in the tip, as shown in Table 1. There is no noticeable difference in the trap density of the firstly ionized Cd vacancy (T2) among all the samples. The trap density of the secondly ionized Cd vacancy (T3) shows a slight decrease from tip to tail. The occupation of vacancy position by In dopant leads to the formation of $\operatorname{In}_{C d}^{+}$: $I n+V_{C d}^{-} \rightarrow \operatorname{In}_{C d}^{+}+2 e$ or $\operatorname{In}+V_{C d}^{2-} \rightarrow \operatorname{In}_{C d}^{+}+3 e \quad[26,27]$. The consequent density of the 
$I n_{C d}^{+}$-related trap (T1) would be lower in the tail than that in the tip.

We employed current-voltage (I-V) analyses and Hall measurements to investigate the material's electrical properties. The compensation between $I n_{C d}^{+}$defects and $\mathrm{Cd}$ vacancies results in low concentration $\left(\sim 5.0 \times 10^{6} \mathrm{~cm}^{-3}\right)$ of net free electrons. High resistivity of $\sim 5.0 \times 10^{10} \Omega \mathrm{cm}$ with n-type conduction at room temperature was obtained from I-V results, as shown in Fig. 3. The Fermi level, acquired by fitting the temperature dependence of bulk resistivity, is pinned near the mid-gap by the $T e_{C d}^{2+}$-related deep donors. Deep-level defects are responsible for high resistivity by stabilizing the compensation conditions against the variations in the concentration of shallow-level defects [10, 28]. CZT crystals with high resistivity can withstand a relatively high bias voltage $(>5000 \mathrm{~V} / \mathrm{cm})$, which will increase the carrier's velocity and improve the detector's performance. As shown in Table 2, Hall mobility for electrons shows a slight decrease from $\sim 467 \mathrm{~cm}^{2} \mathrm{~V}^{-1} \mathrm{~s}^{-1}$ in the tip to $\sim 394 \mathrm{~cm}^{2} \mathrm{~V}^{-1} \mathrm{~s}^{-1}$ in the tail, probably caused by the higher concentration of $T e_{C d}^{2+}$-related deep donors in the tail. Since the deep trap acts as an effective recombination and trapping center, it would strongly affect the carrier transport properties and the consequent detector performances $[8,29]$.

\section{Conclusions}

We utilized SIMPA method to analyze TSC spectra of CZT wafers from the tip, middle, and tail regions of as-grown CdZnTe:In ingots. The results show that the concentration of Te antisite-related deep donors was higher in the tail due to Te enrichment in the melt as solidification proceeds. The concentration of $\mathrm{In}_{\mathrm{Cd}}^{+}$related traps was lower in the tail, and the trap density of Cd vacancies decreased from tip to tail. The compensation between $\mathrm{In}_{C d}^{+}$defects and $\mathrm{Cd}$ vacancies results in low concentration $\left(\sim 5.0 \times 10^{6} \mathrm{~cm}^{-3}\right)$ of net free electrons in the high-resistivity material with n-type 
conduction. Hall mobility for electrons shows a slight decrease from $\sim 467 \mathrm{~cm}^{2} \mathrm{~V}^{-1} \mathrm{~s}^{-1}$ in the tip to $\sim 394$ $\mathrm{cm}^{2} \mathrm{~V}^{-1} \mathrm{~s}^{-1}$ in the tail due to higher concentration of Te antisite-related deep donors in the tail.

\section{Acknowledgements}

This work was supported by the Special Fund of National Key Scientific Instruments and Equipments Development (2011YQ040082), the National 973 Project of China (2011CB610400), the 111 Project of China (B08040), the National Natural Science Foundation of China (NNSFC-61274081, 51372205), the Doctorate Foundation of Northwestern Polytechnical University (CX201102), and Ministry of Education Fund for Doctoral Students Newcomer Awards of China. The authors from BNL acknowledge support from the DOE/NNSA Office of Nuclear Nonproliferation R\&D. The authors would like to give special thanks to Dr. Nianxia Cao at Syracuse University for the great help during the paper writing.

\section{References}

[1] T. Schlesinger, J. Toney, H. Yoon, E. Lee, B. Brunett, L. Franks, R. James, Cadmium zinc telluride and its use as a nuclear radiation detector material, Materials Science and Engineering: R: Reports, 32 (2001) 103-189.

[2] R. James, B. Brunett, J. Heffelfinger, J. Van Scyoc, J. Lund, F. Doty, C. Lingren, R. Olsen, E. Cross, H. Hermon, Material properties of large-volume cadmium zinc telluride crystals and their relationship to nuclear detector performance, Journal of electronic materials, 27 (1998) 788-799.

[3] A. Bolotnikov, G. Camarda, G. Wright, R. James, Factors limiting the performance of CdZnTe detectors, Nuclear Science, IEEE Transactions on, 52 (2005) 589-598.

[4] P. Rudolph, Defect formation during crystal growth from the melt, in: Springer Handbook of Crystal Growth, Springer, 2010, pp. 159-201.

[5] K. Zanio, Cadmium Telluride, Semiconductors and Semimetals, Vol. 13, in, New York: Academic Press, 1978.

[6] A. Castaldini, A. Cavallini, B. Fraboni, P. Fernandez, J. Piqueras, Deep energy levels in CdTe and CdZnTe, Journal of Applied Physics, 83 (1998) 2121-2126.

[7] C. Szeles, CdZnTe and CdTe materials for X - ray and gamma ray radiation detector applications, physica status solidi (b), 241 (2004) 783-790.

[8] M. Chu, S. Terterian, D. Ting, C. Wang, H. Gurgenian, S. Mesropian, Tellurium antisites in CdZnTe, Applied Physics Letters, 79 (2001) 2728-2730.

[9] V. Babentsov, J. Franc, R. James, Compensation and carrier trapping in indium-doped CdTe: Contributions from an important near-mid-gap donor, Applied Physics Letters, 94 (2009) 052102. 
[10] G. Zha, J. Yang, L. Xu, T. Feng, N. Wang, W. Jie, The effects of deep level traps on the electrical properties of semi-insulating CdZnTe, Journal of Applied Physics, 115 (2014) 043715.

[11] D.S. Bale, C. Szeles, Nature of polarization in wide-bandgap semiconductor detectors under high-flux irradiation: Application to semi-insulating Cd 1- x Zn x Te, Physical Review B, 77 (2008) 035205 .

[12] A. Cola, I. Farella, The polarization mechanism in CdTe Schottky detectors, Applied Physics Letters, 94 (2009) 102113.

[13] P. Rudolph, M. Neubert, Defects in CdTe Bridgman monocrystals caused by nonstoichiometric growth conditions, Journal of Crystal Growth, 128 (1993) 582-587.

[14] W. Jie, Principle and Technology of Crystal Growth, Science Press, Beijing, (2010) 110-111.

[15] P. Rudolph, Fundamental studies on Bridgman growth of CdTe, Progress in crystal growth and characterization of materials, 29 (1994) 275-381.

[16] G.A. Dussel, R.H. Bube, Theory of thermally stimulated conductivity in a previously photoexcited crystal, Physical Review, 155 (1967) 764.

[17] Z.Q. Fang, D.C. Look, J. Zhao, Traps in semi - insulating InP studied by thermally stimulated current spectroscopy, Applied Physics Letters, 61 (1992) 589-591.

[18] M. Pavlović, U. Desnica, Precise determination of deep trap signatures and their relative and absolute concentrations in semi-insulating GaAs, Journal of Applied Physics, 84 (1998) 2018-2024.

[19] N. Krsmanovic, K. Lynn, M. Weber, R. Tjossem, T. Gessmann, C. Szeles, E. Eissler, J. Flint, H. Glass, Electrical compensation in CdTe and Cd 0.9 Zn 0.1 Te by intrinsic defects, Physical Review B, 62 (2000) R16279.

[20] E. Lee, R. James, R. Olsen, H. Hermon, Compensation and trapping in CdZnTe radiation detectors studied by thermoelectric emission spectroscopy, thermally stimulated conductivity, and current-voltage measurements, Journal of electronic materials, 28 (1999) 766-773.

[21] L. Xu, W. Jie, G. Zha, Y. Xu, X. Zhao, T. Feng, L. Luo, W. Zhang, R. Nan, T. Wang, Radiation damage on CdZnTe:In crystals under high dose 60Co [gamma]-rays, CrystEngComm, 15 (2013) 10304-10310.

[22] J. Francou, K. Saminadayar, J. Pautrat, Shallow donors in CdTe, Physical Review B, 41 (1990) 12035 .

[23] S.-H. Wei, S. Zhang, Chemical trends of defect formation and doping limit in II-VI semiconductors: The case of CdTe, Physical Review B, 66 (2002) 155211.

[24] A. Carvalho, A. Tagantsev, S. Öberg, P. Briddon, N. Setter, Cation-site intrinsic defects in Zn-doped CdTe, Physical Review B, 81 (2010) 075215.

[25] M. Berding, Native defects in CdTe, Physical Review B, 60 (1999) 8943.

[26] T. Wang, W.Q. Jie, D.M. Zeng, G. Yang, Y.D. Xu, W.H. Liu, J.J. Zhang, Temperature dependence of photoluminescence properties of In-doped cadmium zinc telluride, J. Mater. Res., 23 (2008) 1389-1392.

[27] P. Yu, W. Jie, T. Wang, Detector-grade CdZnTe: In crystals obtained by annealing, Journal of materials science, 46 (2011) 3749-3752.

[28] V. Babentsov, J. Franc, P. Hoeschl, M. Fiederle, K. Benz, N. Sochinskii, E. Dieguez, R. James, Characterization of compensation and trapping in CdTe and CdZnTe: Recent advances, Cryst. Res. Technol., 44 (2009) 1054-1058.

[29] C. Szeles, Advances in the crystal growth and device fabrication technology of CdZnTe room temperature radiation detectors, Nuclear Science, IEEE Transactions on, 51 (2004) 1242-1249. 


\section{Figure captions}

Fig. 1 IR microscopic images and size distribution of Te inclusions in CdZnTe wafers along the growth direction of the as-grown ingots.

Fig. 2 Typical TSC spectra of CdZnTe wafers from the tip, middle, and tail samples of the as-grown ingots.

Table 1 Comparisons of the trap density in CdZnTe wafers from the tip, middle, and tail samples of the as-grown ingots.

Fig. 3 Electrical properties: (a) typical current-voltage (I-V) curve, (b) the Fermi level of CdZnTe crystals from the as-grown ingots.

Table 2 Comparisons of the Hall results in CdZnTe wafers from the tip, middle, and tail samples of the as-grown ingots. 
Fig. 1:
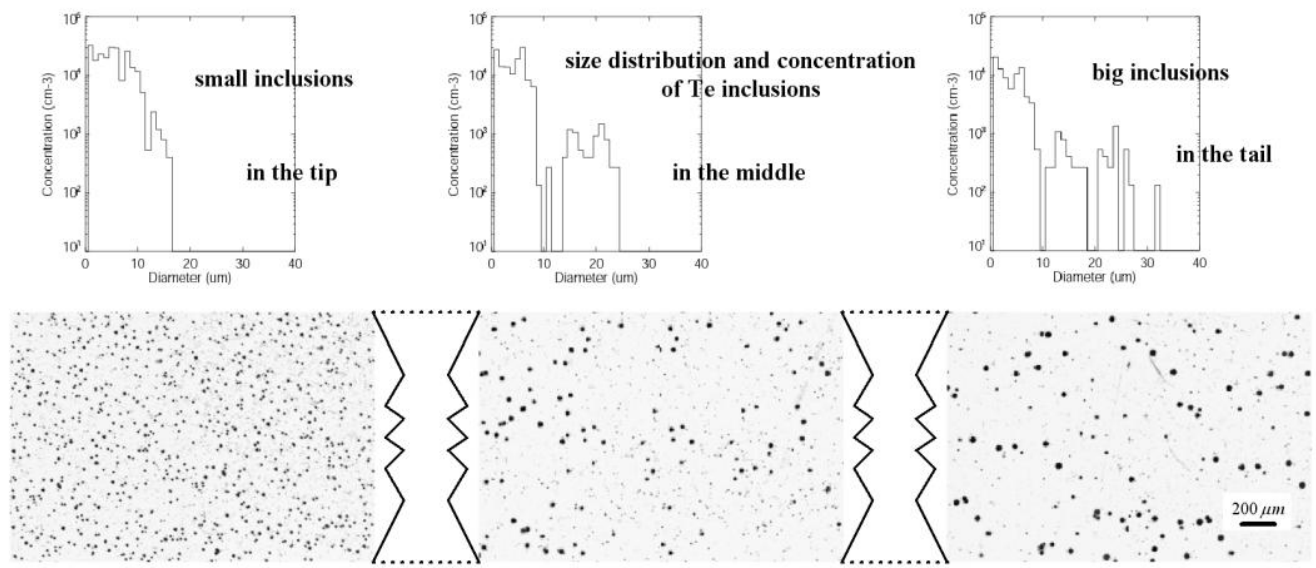

Fig. 2:

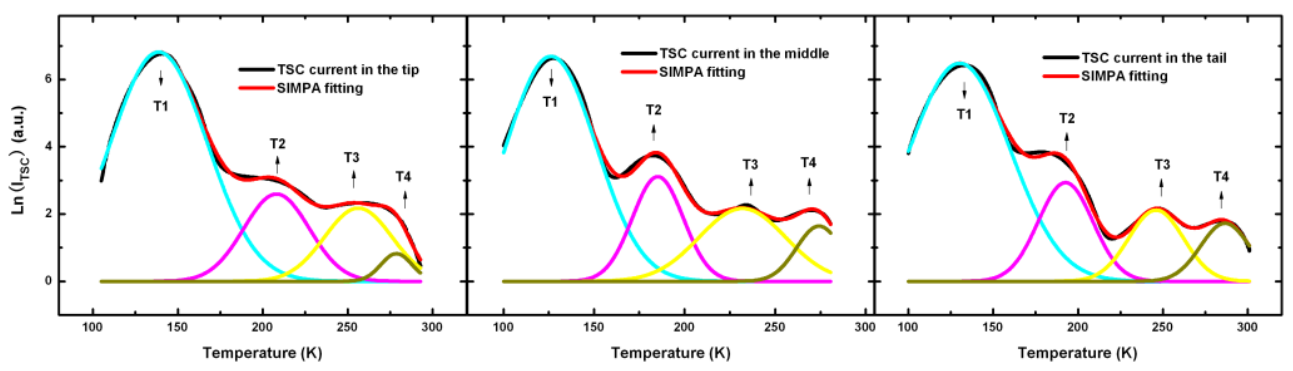

Table 1:

\begin{tabular}{ccccc}
\hline & T1 & T2 & T3 & T4 \\
\hline Tip $\left(\mathrm{cm}^{-3}\right)$ & $1.07 \times 10^{16}$ & $1.20 \times 10^{16}$ & $2.69 \times 10^{15}$ & $2.29 \times 10^{13}$ \\
Middle $\left(\mathrm{cm}^{-3}\right)$ & $8.44 \times 10^{15}$ & $1.30 \times 10^{16}$ & $2.39 \times 10^{15}$ & $7.11 \times 10^{13}$ \\
Tail $\left(\mathrm{cm}^{-3}\right)$ & $8.35 \times 10^{15}$ & $1.27 \times 10^{16}$ & $2.43 \times 10^{15}$ & $7.33 \times 10^{13}$ \\
\hline
\end{tabular}


Fig. 3:

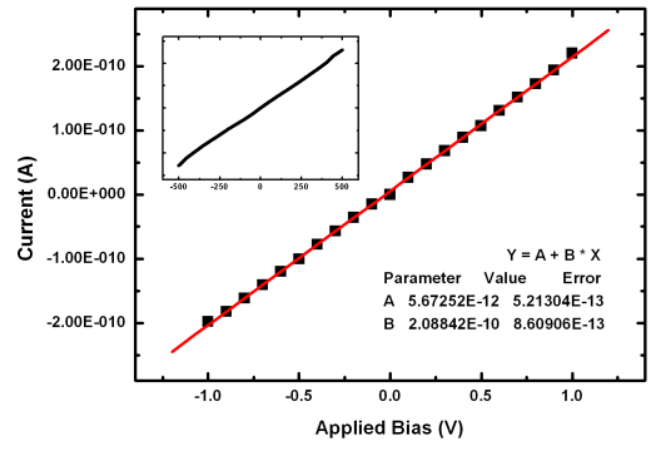

(a)

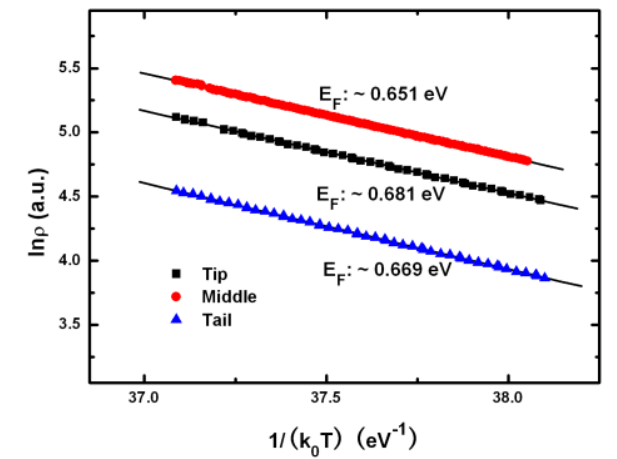

(b)

Table 2:

\begin{tabular}{|c|c|c|c|c|}
\hline & Bulk resistivity $(\Omega \mathrm{cm})$ & Net carrier density $\left(\mathrm{cm}^{-3}\right)$ & Hall mobility $\left(\mathrm{cm}^{2} \mathrm{~V}^{-1} \mathrm{~s}^{-1}\right)$ & Conduction type (n or $\mathrm{p}$ ) \\
\hline Tip & $4.13 \times 10^{9}$ & $3.24 \times 10^{6}$ & 467 & $\mathrm{n}$ \\
\hline Middle & $4.27 \times 10^{9}$ & $3.60 \times 10^{6}$ & 406 & $\mathrm{n}$ \\
\hline Tail & $3.91 \times 10^{9}$ & $4.05 \times 10^{6}$ & 394 & $\mathrm{n}$ \\
\hline
\end{tabular}

